\title{
Neurobiological background of borderline personality disorder, PTSD and ADHD
}

\author{
Andrea Schmitt ${ }^{1} \cdot$ Peter Falkai $^{1}$
}

Published online: 19 February 2016

(C) Springer-Verlag Berlin Heidelberg 2016

Deficits in emotional learning and memory are a core feature in borderline personality disorder (BPD). However, studies investigating the underlying neuronal substrates are lacking. In a functional magnetic resonance imaging (fMRI) study, Krause-Utz et al. [1] investigated 27 medication-free female patients with BPD and 26 female controls with fMRI during a differential delay aversive conditioning paradigm. Besides skin conductance response in subjective ratings, the authors also investigated valence and arousal. They found increased insula activity in BPD patients compared with healthy controls during early acquisition. During extinction, patients had more arousal and activated the amygdala in response to the conditioned stimulus. Amygdala habituation to this stimulus during acquisition was found in healthy controls but not in patients. Altered temporal response patterns with increased vigilance during early acquisition and delayed extinction processes may be a neurobiological background of emotional cognitive symptoms in BPD. In the disorder, genetic and environmental factors may interact and influence response of the hypothalamus-pituitary-adrenal (HPA) axis. In a case-control study, Martín-Blanco et al. [2] for the first time investigated 47 polymorphisms in $10 \mathrm{HPA}$ axis genes and assessed the presence of childhood trauma in a group of 481 patients with BPD and 442 healthy controls. Two polymorphisms in the FKBP5 gene and haplotype combinations of the genes $F K B P 5$ and $C R H R 1$ showed significant associations with BPD. Interestingly, two FKBP5 alleles were more frequent

Andrea Schmitt

Andrea.Schmitt@med.uni-muenchen.de

1 Department of Psychiatry and Psychotherapy, LudwigMaximilians-University Munich, Nußbaumstr. 7, 80336 Munich, Germany in patients with history of physical abuse and emotional neglect and two CRHR2 variants in patients with sexual and physical abuse. Altogether, these results suggest the involvement of genes of the HPA axis and interaction with environmental factors in the pathophysiology of BPD.

Post-traumatic stress disorder (PTSD) and complex PTSD have been proposed as categories of PTSD in the 11th revision of the International classification of diseases (ICD-11). In a 10-year prospective, longitudinal study of a representative community sample of 3021 subjects, Perkonigg et al. [3] investigated associations between class membership, symptom criteria and other mental disorders. They found four distinct classes: Class 1 had episodes with core PTSD symptoms, with $18 \%$ matching the criteria of ICD-11 PTSD, and Class 2 had episodes matched with a high probability of PTSD core symptoms and disturbances in self-organization and $50 \%$ matching with complex PTSD. Class 3 was characterized by episodes with disturbances in self-organization but low probability of PTSD core symptoms. Finally, Class 4 was related to low probability of symptom reports. In summary, findings support the ICD-11 proposal to differentiate between PTSD and complex PTSD. In a neurochemical study, Atli et al. [4] investigated serum malondialdehyde (MDA) as marker of lipid peroxidation and activity of the paraoxonase 1 (PON1) antioxidant enzyme in patients with PTSD after earthquakes, participants who did not develop PTSD after earthquakes and subjects without trauma and earthquake experience. PTSD diagnosis added to the effect of trauma on MDA levels and PON1 activity, and these measures may serve as peripheral biomarkers in future PTSD studies. In patients with psychosis, coercive intervention not only may contribute to alleviated symptoms, but can also induce trauma. Mielau et al. [5] investigated the impact of a personal history of coercive treatment on preferences 
concerning clinical management of patients unable to consent in patients with schizophrenia, schizoaffective disorder, and bipolar disorder plus healthy controls. Patients and controls had different attitudes toward involuntary admission, forced medication, and mechanical restraint. Factors influencing judgement on coercion were subjective experience of coercive interventions, overall functioning, and experience of treatment effectiveness and fairness.

In attention deficit/hyperactivity disorder (ADHD), deficits in task-related attentional engagement have been related to altered attention, default mode, and salience networks. In a resting-state fMRI study in patients with ADHD and healthy controls, Sidlauskaite et al. [6] investigated connectivity between brain regions involved in these circuits. In ADHD, hyperconnectivity was detected between attention networks and within the default and ventral attention network. These alterations may be a basis for deficits in attention abilities in ADHD. In addition, impaired working memory is comprised in ADHD. Salatino-Oliveira et al. [7] examined the association between the MAPIB and NOS1 gene with verbal working memory in children and adolescents with ADHD. Polymorphisms in both genes showed a worse performance in the verbal working memory task. These genes are among the promising genes derived from genome-wide association studies and may be part of an ADHD-related signaling network during neurodevelopment. In ADHD, the stability of symptoms over the lifespan is questionable. Philipp-Wiegmann et al. [8] investigated a large sample of 296 elderly respondents with 11 fulfilling the criteria of adult ADHD and compared them to the control group of 285 probands. The ADHD patients retrospectively reported negative impacts due to ADHD-associated behavior during the lifespan, such as impairments in family life, social relationships, dealing with money, and organization of daily life. Those lifetime stabilities of symptoms may be due to disturbances of neuroplasticity during vulnerable brain periods. According to that hypothesis, Amad et al. [9] propose an age-dependent neuroplasticity framework which implies that a lesion or experience may differentially affect brain plasticity dependent on the timing of the event. This hypothesis is extended to traumatic events in borderline personality disorder and PTSD. Finally, Helmchen [10] addresses ethical concerns in history and contemporary treatment in psychiatry. He proposes to evaluate benefits and risks of medical interventions together with the patient, paying serious attention to her/his wishes plus respect for his contingent dignity and to care for the patient being in need of help. He advocates humanity being the central norm of the physicians' conscience.

\section{References}

1. Krause-Utz A, Keibel-Mauchnik J, Ebner-Priemer U, Bohus M, Schmahl C (2015) Classical conditioning in borderline personality disorder: an fMRI study. Eur Arch Psychiatry Clin Neurosci. doi:10.1007/s00406-015-0593-1

2. Martín-Blanco A, Ferrer M, Soler J, Arranz MJ, Vega D, Calvo N, Elices M, Sanchez-Mora C, García-Martinez I, Salazar J, Carmona C, Bauzà J, Prat M, Pérez V, Pascual JC (2015) The role of hypothalamus-pituitary-adrenal genes and childhood trauma in borderline personality disorder. Eur Arch Psychiatry Clin Neurosci. doi:10.1007/s00406-015-0612-2

3. Perkonigg A, Höfler M, Cloitre M, Wittchen HU, Trautmann S, Maercker A (2015) Evidence for two different ICD-11 posttraumatic stress disorders in a community sample of adolescents and young adults. Eur Arch Psychiatry Clin Neurosci. doi:10.1007/ s00406-015-0639-4

4. Atli A, Bulut M, Bez Y, Kaplan İ, Özdemir PG, Uysal C, Selçuk H, Sir A (2015) Altered lipid peroxidation markers are related to post-traumatic stress disorder (PTSD) and not trauma itself in earthquake survivors. Eur Arch Psychiatry Clin Neurosci. doi:10.1007/s00406-015-0638-5

5. Mielau J, Altunbay J, Gallinat J, Heinz A, Bermpohl F, Lehmann A, Montag C (2015) Subjective experience of coercion in psychiatric care: a study comparing the attitudes of patients and healthy volunteers towards coercive methods and their justification. Eur Arch Psychiatry Clin Neurosci. doi:10.1007/s00406-015-0598-9

6. Sidlauskaite J, Sonuga-Barke E, Roeyers H, Wiersema JR (2015) Altered intrinsic organisation of brain networks implicated in attentional processes in adult attention-deficit/hyperactivity disorder: a resting-state study of attention, default mode and salience network connectivity. Eur Arch Psychiatry Clin Neurosci. doi:10.1007/s00406-015-0630-0

7. Salatino-Oliveira A, Wagner F, Akutagava-Martins GC, Bruxel EM, Genro JP, Zeni C, Kieling C, Polanczyk GV, Rohde LA, Hutz MH (2015) MAP1B and NOS1 genes are associated with working memory in youths with attention-deficit/hyperactivity disorder. Eur Arch Psychiatry Clin Neurosci. doi:10.1007/ s00406-015-0626-9

8. Philipp-Wiegmann F, Retz-Junginger P, Retz W, Rösler M (2015) The intraindividual impact of ADHD on the transition of adulthood to old age. Eur Arch Psychiatry Clin Neurosci. doi:10.1007/s00406-015-0644-7

9. Amad A, Ramoz N, Thomas P, Gorwood P (2015) The agedependent plasticity highlights the conceptual interface between borderline personality disorder and PTSD. Eur Arch Psychiatry Clin Neurosci. doi:10.1007/s00406-015-0648-3

10. Helmchen H (2015) Human dignity and the physician's conscience. Eur Arch Psychiatry Clin Neurosci. doi:10.1007/ s00406-015-0635-8 